\title{
Functional Dependence between the Hamiltonian and the Modular Operator Associated with a Faithful Invariant State of a $W^{*}$-Dynamical System
}

\author{
By
}

\author{
J. DE CANNIÈRE*)
}

\begin{abstract}
Let $H$ and $\Delta$ be the hamiltonian, resp. the modular operator associated with an invariant faithful normal state $\omega$ of a $W^{*}$-dynamical system $(A, \alpha)$. Then $\Delta=f(H)$ for some decreasing function $f$ if and only if (roughly speaking) $\omega$ is 2-passive with respect to $\alpha$. It follows that under certain conditions a 3-passive state is an equilibrium (i.e. KMS) state.
\end{abstract}

\section{$\S 1$. Introduction and Basic Definitions}

In this paper, as in an earlier one [7], we investigate certain spectral properties of invariant states of noncommutative dynamical systems closely related to the KMS condition.

Let $\mathscr{A}$ be a von Neumann algebra, and $\alpha=\left\{\alpha_{t}\right\}(t \in \mathbb{R})$ an ultra

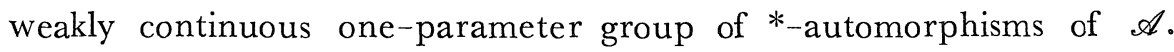
The action $\alpha$ of $\boldsymbol{R}$ on $\mathscr{A}$ can be described by the "spectral resolution" of $\mathscr{A}$ it induces. Let us recall that each closed interval $[\lambda, \mu]$ in $\mathbb{R}$ determines a spectral subspace $M[\lambda, \mu]$, a generic element $x$ of which is characterized by the property that $\int f(t) \alpha_{t}(x) d t=0$ whenever $f \in L^{1}(\boldsymbol{R})$ and the (inverse) Fourier transform $\hat{f}$ of $f$ is supported in the complement of $[\lambda, \mu]$ [3, Definition 2.1 and Remark on p. 225]. Here $\hat{f}$ is defined by

$$
\hat{f}(\lambda)=\int e^{i \lambda t} f(t) d t \quad(\lambda \in \boldsymbol{R}) .
$$

Communicated by H. Araki, September 25, 1982.

*) On leave from N. F. W. O. and K. U. Leuven, Belgium.

Department of Mathematics, University of California, Berkeley, CA 94720, USA. 
It is also useful to consider the ultraweakly closed linear span $R(\lambda, \mu)$ of the set of all elements of $\mathscr{A}$ of the form $\int f(t) \alpha_{t}(y) d t$, where $y \in \mathscr{A}, f \in L^{1}(\boldsymbol{R})$ and the support of $\hat{f}$ is contained in the open interval $(\lambda, \mu)$ [11, Definition 2.3.2] (clearly $R(\lambda, \mu) \subset M[\lambda, \mu]$ ). The knowledge of the collection of all the $M[\lambda,+\infty)$, or all the $R(\lambda$, $+\infty$ ) (which we call a spectral resolution of $\mathscr{A}$ ), is equivalent with the knowledge of $\alpha$, at least in principle [3, Lemma 2 on p. 233].

In [7, Definition 1.2] we introduced the notion of spectral passivity:

Definition 1.1. An $\alpha$-invariant normal state $\omega$ of $\mathscr{A}$ is said to be $n$-spectrally passive (where $n$ is a positive integer) if

$$
\prod_{i=1}^{n} \omega\left(x_{i} x_{i}^{*}\right) \leq \prod_{i=1}^{n} \omega\left(x_{i}^{*} x_{i}\right)
$$

for all $n$-tuples $x_{1}, x_{2}, \ldots, x_{n}$ of elements of $\mathscr{A}$ such that $x_{i} \in R\left(\lambda_{i}\right.$, $+\infty)(i=1,2, \ldots, n)$ for some $n$-tuple $\lambda_{1}, \lambda_{2}, \ldots, \lambda_{n}$ of real numbers satisfying $\sum_{i=1}^{n} \lambda_{i} \geq 0$. The term "1-spectral passivity" will be discarded in favour of "spectral passivity".

The interest of this notion lies in its relationship with the concept of passivity introduced by Pusz and Woronowicz $[12 ; 7$, Theorem 3. 3], but also appears immediately in view of the following formulation of the KMS condition, obtained in [7]: $\omega$ is $\beta$-KMS with respect to $\alpha$ (where $0 \leq \beta \leq+\infty$ ) if and only if it is $\alpha$-invariant and

$$
x \in R(\lambda,+\infty) \Rightarrow \omega\left(x x^{*}\right) \leq e^{-\beta \lambda} \omega\left(x^{*} x\right)
$$

[7, Theorem 1.1 and Remark 2.1(i)].

It is clear, then, that a $\beta$-KMS state is $n$-spectrally passive for all $n$ (i. e. completely spectrally passive $[7$, Definition 1.1]), whatever the value of $\beta$. Quite remarkably the converse is true as well: a completely spectrally passive state is in fact a KMS state for some $\beta$. In this form, this was stated and proved in [7], but it already appears implicitly as Theorem 1.4 in [12]. Recently, Batty provided a truly elementary procf, with (1) as a starting point [4, Section 2].

The argument developed in [7] retains some interest, however, for two reasons. On the one hand it was generalized in a significant way by Batty, who obtained important results concerning the existence of one-parameter subgroups of arbitrary abelian groups acting on a 
$C^{*}$-algebra for which a given invariant state is KMS [5, Theorem 3.2]. On the other hand, the constructions of [7, Section 4] can be used to analyze in detail the mechanism by which complete spectral passivity forces a state to be KMS. This is the purpose of the present paper. In particular it will be shown that 2-spectral passivity (or rather a naturally arising stronger version of it) already imposes so much structure on the triple $(\mathscr{A}, \alpha, \omega)$ that it implies the existence of a decreasing real function $f(\lambda)$ on the "energy spectrum" of the system generalizing, in a certain sense, the Boltzmann factor $e^{-\beta \lambda}$.

We shall have to make this statement precise. But at this point the reader should be warned that the setup is somewhat different from the one in [7]. Indeed we assume that $(\mathscr{A}, \alpha)$ is a $W^{*}$-dynamical system rather than a $C^{*}$-dynamical system. Moreover we suppose throughout the paper (except in Remark 2.13) that $\omega$ is a faithful normal $\alpha$-invariant state of $\mathscr{A}$. Consequently we can consider $\mathscr{A}$ to act on a Hilbert space $\mathscr{H}$ with cyclic and separating vector $\Omega$ such that $\omega(x)=(\Omega, x \Omega) \quad(x \in \mathscr{A})$. We also know that there is a unique strongly continuous one-parameter group $U=\left\{U_{t}\right\}(t \in \boldsymbol{R})$ of unitaries implementing $\alpha$ and leaving $\Omega$ invariant. The hamiltonian of the system is the self-adjoint operator $H$ on $\mathscr{H}$ defined by the equation $U_{t}=e^{i t H}(t \in \boldsymbol{R})$.

As a consequence of our faithfulness assumption the TomitaTakesaki theory is available to us [13]. Let $\Delta$ be the modular operator associated with $\Omega$. In general, the self-adjoint operators $\Delta$ and $H$ are not related in any particular way, except for the fact that they commute strongly. On the other hand, as is well known, $\omega$ is $\beta$-KMS (with $0 \leq \beta<+\infty$ ) if and only if $\Lambda=e^{-\beta H}$. Suppose more generally that $\omega$ is 2-spectrally passive. The main result of this paper (Theorem 2. 8) asserts that, under certain additional hypotheses, there exists a decreasing positive function $f$ on the spectrum $\sigma(H)$ of $H$ such that $\Delta=f(H)^{*)}$. Conversely, the existence of such a function implies that $\omega$ is 2-spectrally passive (Theorem 2.12). If in fact $\omega$ satisfies the 3 -spectral passivity condition, and if moreover $\sigma(H)=\boldsymbol{R}$, then the function $f$ turns out to be a decreasing exponential, so that $\omega$ is KMS (Theorem 3.2).

*) In fact this still holds, in a somewhat generalized sense, when $\omega$ is not faithful. Cf. Remark 2. 13.

**) This result was first obtained by H. A. M. Daniëls in [6b]. Cf. Remark 3. 3. 
Having spelled out the principal results in sufficient detail, we would now like to present a motivation for the stronger form of the 2-spectral passivity condition needed to obtain them. To that end we consider the simple case where $\mathscr{A}=\mathscr{L}(\mathscr{S})$ with $\mathfrak{S} \cong \mathbb{C}^{q}$. In this case the dynamical group $\alpha$ is determined by a hermitian operator $h$ on $\mathfrak{S}$ via the formula $\alpha_{t}(x)=e^{i h t} x e^{-i h t}(x \in \mathscr{L}(\mathfrak{S}), t \in \boldsymbol{R})$, whereas a faithful $\alpha$-invariant state $\omega$ is given by a positive invertible operator $\rho$, commuting with $h$, such that $\omega(x)=\tau(\rho x)$ for all $x$ in $\mathscr{L}(\mathfrak{S})$ (here $\tau$ denotes the trace on $\mathscr{L}(\mathfrak{S})$ ).

Let $\left\{\phi_{j}\right\}_{j=1}^{q}$ be an orthonormal basis of $\mathfrak{S}$ in which both $h$ and $\rho$ are diagonal. Let $h_{1}, h_{2}, \ldots, h_{q}$ and $\rho_{1}, \rho_{2}, \ldots, \rho_{q}$ be the corresponding eigenvalues of $h$ and $\rho$, respectively. It is not difficult to show that $\omega$ is 2-spectrally passive with respect to $\alpha$ if and only if the following holds [7, Example 4.9]:

$$
\left\{\begin{array}{l}
\text { whenever } h_{j}-h_{k}+h_{j^{\prime}}-h_{k^{\prime}}>0 \\
\quad \text { for some } j, k, j^{\prime}, k^{\prime} \in\{1,2, \ldots, q\}, \\
\text { one has } \rho_{j} \rho_{j^{\prime}} \leq \rho_{k} \rho_{k^{\prime}} .
\end{array}\right.
$$

In order to determine the implications of the condition (2) on the modular structure induced by $\omega$, we have to identify $\mathscr{A}$ with $\mathscr{A}$ $\otimes 1$ acting on $\mathscr{H}=\mathfrak{S} \otimes \widetilde{\mathscr{S}}$, and to put $\Omega=\sum_{j=1}^{q} \rho_{j}^{1 / 2} \phi_{j} \otimes \phi_{j}$. One easily computes that $H=h \otimes 1-1 \otimes h$ and that $\Delta=\rho \otimes \rho^{-1}$. Both $H$ and $\Delta$ are clearly diagonal in the basis $\left\{\phi_{j} \otimes \phi_{k}\right\}_{j, k=1}^{q}$, and the corresponding eigenvalues are $h_{j}-h_{k}$ (for $H$ ) and $\rho_{j} \rho_{k}^{-1}$ (for $\Delta$ ). Hence if we replace (2) with the stronger requirement that

$$
h_{j}-h_{k}+h_{j^{\prime}}-h_{k^{\prime}} \geq 0 \Rightarrow \rho_{j} \rho_{j^{\prime}} \leq \rho_{k} \rho_{k^{\prime}},
$$

we can unambiguously define a decreasing function $f$ on $\sigma(H)$ by $f\left(h_{j}-h_{k}\right)=\rho_{j} \rho_{k}^{-1}$, so that $f(H)=\Delta$. Clearly this conclusion could not have been obtained in general without some strengthening of (2).

Passing from (2) to (3) really amounts to replacing the spectral subspaces $R(\lambda,+\infty)$ in Definition 1.1 by the larger spaces $M[\lambda,+\infty)$ corresponding to the closed half lines $[\lambda,+\infty)$. Hence we are led to introduce the following definition in the general case:

Definition 1. 2. An $\alpha$-invariant normal state $\omega$ of $\mathscr{A}$ is called strongly $n$-spectrally passive if $x_{i} \in M\left[\lambda_{i},+\infty\right)(i=1,2, \ldots, n)$ implies $\prod_{i=1}^{n} \omega\left(x_{i} x_{i}^{*}\right) \leq \prod_{i=1}^{n} \omega\left(x_{i}^{*} x_{i}\right)$ whenever $\sum_{i=1}^{n} \lambda_{i} \geq 0$. 
Is it possible to justify the substitution of strong spectral passivity for spectral passivity on physical grounds? In the above example of a "finite spin system" the answer seems to be affirmative, in view of Lenard's analysis in [10] of what he calls "structural stability". Extrapolating from the finite dimensional case one may conjecture that strong spectral passivity should result from a combination of spectral passivity and stability in the sense of Haag, Kastler and Trych-Pohlmeyer [9]. We give some substance to that claim in Lemma 2.10 and Remark 2.11. Another question is, whether strong spectral passivity implies passivity in the original sense of Pusz and Woronowicz [12]. This is by no means clear a priori (the converse is false).

We now return briefly to the case $\mathscr{A}=\mathscr{L}(\mathfrak{S})$ considered above to make a different point. In this case strong spectral passivity reduces to the condition that $h_{j}-h_{k} \geq 0$ implies $\rho_{j} \leq \rho_{k}$, which means exactly that $\rho$ is a decreasing function of $h$. For general quantum statistical systems there is no density matrix, of course. However we are able to derive a formally analogous relation $\Delta=f(H)$, with $\Delta$ in place cf $\rho$ and $h$ replaced by $H$ (the spectrum of which consists of energy differences rather than energy levels). Thus our result provides an illustration of the heuristic principle that the modular operator $\Delta$ can be used as a partial substitute for the density matrix $\rho$ in the general case, albeit in a "relative" rather than in an "absolute" sense. In some of Araki's papers (e.g. [1,2]), which inspired us, the same philosophy is at work.

It is unclear at present what physical meaning (if any) should be attributed to the relation $\Delta=f(H)^{*}$. By comparison with the exponentials $e^{-\beta \lambda}$, the function $f(\lambda)$ can conceivably provide us with a quantitative measure of the deviation of the state $\omega$ from equilibrium. More specifically one might expect $\log f$, or some quantity derived from it, to have certain entropy-like properties (indeed passivity is an expression of the second law of thermodynamics [12], which itself gives rise to the notion of entropy).

Finally it is worthwhile observing that a decreasing dependence

* The same type of relation appears in a recent paper by J. S. Cohen, H. A. M. Daniëls and M. Winnink [Commun. Math. Phys., 84, 449-458 (1982)], where it is studied as a consequence of a modified KMS equation. I am grateful to the authors for pointing this out to me. 
between passive states and hamiltonians has also been shown to exist in the framework of classical statistical mechanics [8, Proposition 2 ; 6a, Theorem 1;6b, Theorem 6.2].

\section{§2. Proof of $\Delta=f(H)$}

We assume throughout the paper that $\mathscr{A}$ is a ( $\sigma$-finite) von Neumann algebra acting on a Hilbert space $\mathscr{H}$ with cyclic and separating vector $\Omega$. Furthermore $\left\{U_{t}\right\}(t \in \boldsymbol{R})$ is a strongly continuous one-parameter group of unitary operators on $\mathscr{H}$ satisfying $U_{t} \Omega=\Omega$ and $U_{t} \mathscr{A} U_{t}^{-1}=\mathscr{A}$. The state of $\mathscr{A}$ defined by $\Omega$ is denoted $\omega$, the restriction of Ad $U_{t}$ to $\mathscr{A}$ is called $\alpha_{t}$, and $U_{t}=e^{i t H}(t \in \boldsymbol{R})$.

Let $P$ be the unique projection-valued measure such that $H=$ $\int \lambda d P_{\lambda}$. For every $x$ in $\mathscr{A}$ we define bounded positive Radon measures $\mu_{x}$ and $\nu_{x}$ on $\boldsymbol{R}$ by their (inverse) Fourier transforms:

$$
\left\{\begin{aligned}
\omega\left(x^{*} \alpha_{t}(x)\right) & =\int e^{i \lambda t} d \mu_{x}(\lambda) \\
\text { and } & \\
\omega\left(\alpha_{t}(x) x^{*}\right) & =\int e^{i \lambda t} d \nu_{x}(\lambda) \quad(t \in \boldsymbol{R}) .
\end{aligned}\right.
$$

Equivalently we have

$$
\left\{\begin{aligned}
d \mu_{x}(\lambda) & =d\left(x \Omega, P_{\lambda} x \Omega\right) \\
\text { and } & \\
d \nu_{x}(\lambda) & =d\left(x^{*} \Omega, P_{-\lambda} x^{*} \Omega\right) .
\end{aligned}\right.
$$

The following proposition gives a simple but very useful reformulation of the relation $\Delta=f(H)$ in terms of the measures $\mu_{x}$ and $\nu_{x}$.

Proposition 2.1. Let $f: \boldsymbol{R} \rightarrow[0,+\infty]$ be Borel measurable and $P$-almost everywhere finite. Then the following statements are equivalent :

(i) $\Delta=f(H)$.

(ii) For all $x$ in $\mathscr{A}, \nu_{x}$ is absolutely continuous with respect to $\mu_{x}$, and $\frac{d \nu_{x}}{d \mu_{x}}=f$ ( $\mu_{x}$-almost everywhere $)$.

*) Except, as pointed out previously, in Remark 2. 13. 
Proof. (i) $\Rightarrow$ (ii). Suppose $\Delta=f(H)$ and $x \in \mathscr{A}$. For $g$ in $L^{1}(\boldsymbol{R})$ we define $y=\int g(t) \alpha_{t}(x) d t$. It is easy to see that $d \mu_{y}(\lambda)=|\hat{g}(\lambda)|^{2} d \mu_{x}(\lambda)$ and $d \nu_{y}(\lambda)=|\hat{g}(\lambda)|^{2} d \nu_{x}(\lambda)$. Using this we have

$$
\begin{aligned}
\int|\hat{g}(\lambda)|^{2} d \nu_{x}(\lambda) & =\omega\left(y y^{*}\right) \text { by }(4) \\
& =\left\|y^{*} \Omega\right\|^{2}=\left\|\Delta^{1 / 2} y \Omega\right\|^{2} \\
& =\left\|f(H)^{1 / 2} y \Omega\right\|^{2} \text { by hypothesis } \\
& =\int f(\lambda) d \mu_{y}(\lambda) \text { by }(5) \\
& =\int|\hat{g}(\lambda)|^{2} f(\lambda) d \mu_{x}(\lambda) .
\end{aligned}
$$

As $x \Omega \in \operatorname{dom} \Delta^{1 / 2}=\operatorname{dom} f(H)^{1 / 2}$, it follows that $f \in L^{1}\left(\mu_{x}\right)$. Hence by uniform density of $L^{1}(\boldsymbol{R})^{\wedge}$ in $C_{0}(\boldsymbol{R})$ we actually obtain

$$
\int h(\lambda) d \nu_{x}(\lambda)=\int h(\lambda) f(\lambda) d \mu_{x}(\lambda)
$$

for all nonnegative $h$ in $C_{0}(\boldsymbol{R})$. This yields (ii) by the LebesgueRadon-Nikodým theorem.

(ii) $\Rightarrow$ (i). We assume that $\nu_{x} \ll \mu_{x}$ and $\frac{d \nu_{x}}{d \mu_{x}}=f$ for all $x$ in $\mathscr{A}$. Since $f \in L^{1}\left(\mu_{x}\right)$ by the boundedness of $\nu_{x}$, it follows that $x \Omega \in$ $\operatorname{dom} f(H)^{1 / 2}$ and

$$
\left\|f(H)^{1 / 2} x \Omega\right\|^{2}=\int f(\lambda) d \mu_{x}(\lambda)=\int d \nu_{x}(\lambda)=\left\|x^{*} \Omega\right\|^{2}=\left\|d^{1 / 2} x \Omega\right\|^{2} .
$$

As $\{x \Omega \mid x \in \mathscr{A}\}$ is a core for $\Delta^{1 / 2}$, the above equation easily implies that $\operatorname{dom} \Delta^{1 / 2} \subset \operatorname{dom} f(H)^{1 / 2}$, and that $\left\|\Delta^{1 / 2} \Phi\right\|=\left\|f(H)^{1 / 2} \Phi\right\|$ for all $\Phi$ in $\operatorname{dom} \Delta^{1 / 2}$. For the remainder of the procf we set $f(H)=A$, and show the following:

2.2. Let $\Delta$ and $A$ be strongly commuting positive sclfadjoint operators on a Hilbert space $\mathscr{H}$ such that $\operatorname{dom}{\Delta^{1 / 2}} \subset \operatorname{dom} A^{1 / 2}$ and

$$
\Phi \in \operatorname{dom} \Delta^{1 / 2} \Rightarrow\left\|\Delta^{1 / 2} \Phi\right\|=\left\|A^{1 / 2} \Phi\right\| . \quad \text { Then } \Delta=A .
$$

First we prove that $\operatorname{dom} A^{1 / 2} \subset \operatorname{dom} d^{1 / 2}$. So suppose $\Psi \in \operatorname{dom} A^{1 / 2}$, and let $E_{n}$ be the spectral projection of $\Delta^{1 / 2}$ corresponding to the interval $[0, n](n \in N)$. As $\Delta^{1 / 2}$ commutes strongly with $A^{1 / 2}$, one has $E_{n} \Psi \in$ dom $A^{1 / 2}$ and $A^{1 / 2} E_{n} \Psi=E_{n} A^{1 / 2} \Psi$. But on the other hand $E_{n} \Psi \in$ dom $\Delta^{1 / 2}$, and 


$$
\begin{aligned}
\left\|\Delta^{1 / 2} E_{n} \Psi-\Delta^{1 / 2} E_{m} \Psi\right\| & =\left\|A^{1 / 2} E_{n} \Psi-A^{1 / 2} E_{m} \Psi\right\| \quad \text { by assumption, } \\
& =\left\|\left(E_{n}-E_{m}\right) A^{1 / 2} \Psi\right\|,
\end{aligned}
$$

which tends to zero as $n, m \rightarrow \infty$. As $\Delta^{1 / 2}$ is closed, we conclude that $\Psi=\lim _{n \rightarrow \infty} E_{n} \Psi \in \operatorname{dom} \Delta^{1 / 2}$.

Finally, take $\Phi \in \operatorname{dom} \Delta$ and $\Psi \in \operatorname{dom} A^{1 / 2}$. Then $\Phi \in \operatorname{dom} \Delta^{1 / 2}=$ $\operatorname{dom} A^{1 / 2}$ and

$$
\begin{aligned}
\left(A^{1 / 2} \Phi, A^{1 / 2} \Psi\right) & =\left(\Delta^{1 / 2} \Phi, \Delta^{1 / 2} \Psi\right) \quad \text { by polarization } \\
& =(\Delta \Phi, \Psi) .
\end{aligned}
$$

It follows that $A^{1 / 2} \Phi \in \operatorname{dom} A^{1 / 2}$, hence $\Phi \in \operatorname{dom} A$, and that $A \Phi=$ $A^{1 / 2}\left(A^{1 / 2} \Phi\right)=\Delta \Phi$. Consequently $\Delta \subset A$. By selfadjointness $A \subset \Delta$. This ends the proof of both (2.2) and Proposition 2.1.

Next we define the function $f$ that appears in the relation $\Delta=$ $f(H)$, as well as an auxiliary function $g$ (these are similar, but not equal to objects with the same name defined in [7]).

Definition 2.3. For $\lambda$ in $\boldsymbol{R}$ we put

$$
\begin{aligned}
& f(\lambda)=\sup \left\{\|x * \Omega\|^{2}\|x \Omega\|^{-2} \mid x \in M[\lambda,+\infty), x \neq 0\right\} \\
& g(\lambda)=\inf \left\{\left\|x^{*} \Omega\right\|^{2}\|x \Omega\|^{-2} \mid x \in M(-\infty, \lambda], x \neq 0\right\},
\end{aligned}
$$

with the convention that $\sup \phi=0$, inf $\phi=+\infty$.

The following lemma will be used to study the properties of the functions $f$ and $g$ (Lemma 2.5 below), and again in the proofs of the lemmas 2.7 and 2. 10 .

Lemma 2.4. If $x \in \mathscr{A}$ and $\lambda \in \boldsymbol{R}$, then there exist $y$ and $z$ in $\mathscr{A}$ such that

(i) $x=y+z, y \in M(\{\lambda\}), z \in R(\boldsymbol{R} \backslash\{\lambda\})$.

If $x \in M[\lambda,+\infty)$, then $z$ can be chosen in $R(\lambda,+\infty)$. Moreover, if (i) holds, then one also has

(ii) $P(\{\lambda\}) x \Omega=y \Omega, P(\{-\lambda\}) x^{*} \Omega=y^{*} \Omega$, and

(iii) $\lim _{T \rightarrow \infty} \frac{1}{2 T} \int_{-T}^{T} e^{-i \lambda t} \omega\left(x^{*} \alpha_{t}(x)\right) d t=\|y \Omega\|^{2}$,

$$
\lim _{T \rightarrow \infty} \frac{1}{2 T} \int_{-T}^{T} e^{-i \lambda t} \omega\left(\alpha_{t}(x) x^{*}\right) d t=\left\|y^{*} \Omega\right\|^{2} .
$$


Proof. Let $f$ in $L^{1}(\boldsymbol{R})$ be such that $\hat{f}(\xi)=1$ if $|\xi| \leq 1, \hat{f}(\xi)=0$ if $|\xi| \geq 2$. For $n=1,2, \ldots$, define $f_{n}$ by

$$
\begin{aligned}
& f_{n}(t)=e^{-i \lambda t} n^{-1} f\left(n^{-1} t\right) \quad(t \in \mathbb{R}), \\
& \text { i. e. } \quad \dot{f}_{n}(\xi)=\hat{f}(n(\xi-\lambda)) \quad(\xi \in \mathbb{R}) \text {. }
\end{aligned}
$$

Put $y_{n}=\int f_{n}(t) \alpha_{t}(x) d t$ and $z_{n}=x-y_{n}$. Then $y_{n} \in R\left(\lambda-3 n^{-1}, \lambda+3 n^{-1}\right)$ and $z_{n} \in M\left(\boldsymbol{R} \backslash\left(\lambda-n^{-1}, \lambda+n^{-1}\right)\right)$. If $x \in M[\lambda,+\infty)$ then in fact $z_{n} \in$ $M\left[\lambda+n^{-1},+\infty\right)$.

Since $\left\|y_{n}\right\| \leq\|f\|_{1}\|x\|$, the ultraweak compactness of the unit ball of $\mathscr{A}$ implies that the sequence $\left\{y_{n}\right\}_{n=1}^{\infty}$ has an ultraweak accumulation point $y$, which belongs to $M(\{\lambda\})$. Since $x-y$ is an accumulation point of $\left\{z_{n}\right\}_{n=1}^{\infty}$, it is an element of $R(\boldsymbol{R} \backslash\{\lambda\})$, and even of $R(\lambda,+\infty)$ if $x \in M[\lambda,+\infty)$. This concludes the proof of (i).

To show (ii) it is sufficient to observe that $y \Omega \in P(\{\lambda\}) \mathscr{H}, y^{*} \Omega \in$ $P(\{-\lambda\}) \mathscr{H}, z \Omega \in P(\boldsymbol{R} \backslash\{\lambda\}) \mathscr{H}$ and $z^{*} \Omega \in P(\mathbb{R} \backslash\{-\lambda\}) \mathscr{H} \quad$ [7, Lemma 1.4].

Finally, according to the mean ergodic theorem,

$$
\begin{aligned}
\lim _{T \rightarrow \infty} \frac{1}{2 T} \int_{-T}^{T} e^{-i \lambda t} \omega\left(x^{*} \alpha_{t}(x)\right) d t & =\lim _{T \rightarrow \infty} \frac{1}{2 T} \int_{-T}^{T} e^{-i \lambda t}\left(x \Omega, U_{t} x \Omega\right) d t \\
& =(x \Omega, P(\{\lambda\}) x \Omega),
\end{aligned}
$$

and similarly

$$
\begin{aligned}
\lim _{T \rightarrow \infty} \frac{1}{2 T} \int_{-T}^{T} e^{-i \lambda t} \boldsymbol{\omega}\left(\alpha_{t}(x) x^{*}\right) d t & =\lim _{T \rightarrow \infty} \frac{1}{2 T} \int_{-T}^{T} e^{-i \lambda t}\left(x^{*} \Omega, U_{-t} x^{*} \Omega\right) d t \\
& =\left(x^{*} \Omega, P(\{-\lambda\}) x^{*} \Omega\right) .
\end{aligned}
$$

Then (iii) follows from (ii).

We note that the existence of a cyclic and separating vector is not required for the validity of (i) above. On the other hand the decomposition $x=y+z$ need not be unique. For instance, if $\mathscr{A}=L^{\infty}(\boldsymbol{R})$ and $\alpha$ is the group of translations, then the functions $\mathrm{t} \longmapsto e^{i \lambda t}$ belong to $R(\boldsymbol{R} \backslash\{0\})$ if $\lambda \neq 0$, but they tend ultraweakly to the constant function 1 when $\lambda \rightarrow 0$. Hence $1 \in M(\{0\}) \cap R(\boldsymbol{R} \backslash\{0\})$.

\section{Lemma 2.5.}

(i) $f$ and $g$ are decreasing functions.

(ii) $f(-\lambda)=g(\lambda)^{-1}$ for all $\lambda$ in $\boldsymbol{R}$ (with $\left.0^{-1}=+\infty,(+\infty)^{-1}=0\right)$. 
(iii) $f(0) \geq 1, g(0) \leq 1$.

(iv) $f(\lambda)=0$ (or, equivalently, $g(-\lambda)=+\infty$ ) if and only if $\sigma(H) \subset[-\lambda, \lambda]$ and $\pm \lambda$ is not an eigenvalue of $H$.

(v) If $\lambda \in \sigma(H)$ then $g(\lambda+) \leq f(\lambda-)^{*}$.

Proof. (i), (ii) and (iii) are obvious. Suppose $f(\lambda)=0$. By (i) and (iii), $\lambda>0$. The definition of $f$ implies $M[\lambda,+\infty)=\{0\}$. In particular $R(\lambda,+\infty)=\{0\}$, hence $\sigma(H)=\operatorname{sp}(\alpha) \subset(-\infty, \lambda]$ [7, Remark 1.5]. But since $s p(\alpha)=-s p(\alpha)$, we actually have the inclusion $\sigma(H) \subset[-\lambda, \lambda]$. Furthermore $M(\{\lambda\})=\{0\}$. As $\Omega$ is cyclic, Lemma 2.4 implies $P(\{ \pm \lambda\})=0$, i. e. $\pm \lambda$ is not an eigenvalue of $H$. Suppose conversely that $\sigma(H) \subset[-\lambda, \lambda]$ and that $\pm \lambda$ is not an eigenvalue of $H$. If $x \in M[\lambda,+\infty)$, then as $\Omega$ is separating $s p(x) \subset[-\lambda, \lambda] \cap[\lambda$, $+\infty)=\{\lambda\} \quad[7$, Remark 1.5]. But the fact that $\lambda$ is not an eigenvalue of $H$ clearly implies $M(\{\lambda\})=\{0\}$. Hence $M[\lambda,+\infty)=\{0\}$ and $f(\lambda)=0$. This ends the procf of (iv). To show (v), suppose $\lambda \in \sigma(H)$ and $\varepsilon>0$. Then we can find a nonzero element $x$ in $M[\lambda-\varepsilon, \lambda+\varepsilon]$. It follows that $g(\lambda+\varepsilon) \leq\left\|x^{*} \Omega\right\|^{2}\|x \Omega\|^{-2} \leq f(\lambda-\varepsilon)$. Taking the limit as $\varepsilon \rightarrow 0$ gives the desired result.

Next we show how to express the passivity of $\omega$ in terms of $f$ and $g$.

Lemma 2.6. (i) $\omega$ is strongly spectrally passive if and only if $f(0) \leq 1$ (or equivalently, $g(0) \geq 1$ ).

(ii) $\omega$ is strongly 2-spectrally passive if and only if $f(\lambda) \leq g(\lambda)(\lambda \in \boldsymbol{R})$.

Proof. (i) is obvious (if $\omega$ is strongly spectrally passive one actually has $f(0)=g(0)=1$, by Lemma 2.5 (iii)).

(ii) Suppose that $\omega$ is strongly 2 -spectrally passive, $\lambda \in \boldsymbol{R}$, and $f(\lambda)>0$, $g(\lambda)<+\infty$. For every nonzero $x$ in $M[\lambda,+\infty)$ and nonzero $y$ in $M(-\infty, \lambda]$, Definition 1.2 implies that

$$
\omega\left(x x^{*}\right) \omega\left(y^{*} y\right) \leq \omega\left(x^{*} x\right) \omega\left(y y^{*}\right),
$$

or

$$
\omega\left(x x^{*}\right) \omega\left(x^{*} x\right)^{-1} \leq \omega\left(y y^{*}\right) \omega\left(y^{*} y\right)^{-1} .
$$

*) $g(\lambda+)=\lim _{\substack{>>\lambda \\ \mu \rightarrow \lambda}} g(\mu), f(\lambda-)=\lim _{\substack{<\rightarrow \lambda \\ \mu \rightarrow \lambda}} f(\mu)$. 
It follows that $f(\lambda) \leq g(\lambda)$. If $f(\lambda)=0$ or $g(\lambda)=+\infty$ this inequality holds trivially. Conversely, if $0<f(\lambda) \leq g(\lambda)<+\infty, x \in M[\lambda,+\infty)$, $z \in M[-\lambda,+\infty)$, then

$$
\omega\left(x x^{*}\right) \leq f(\lambda) \omega\left(x^{*} x\right), \omega\left(z z^{*}\right) \leq g(\lambda)^{-1} \omega\left(z^{*} z\right) .
$$

As $f(\lambda) g(\lambda)^{-1} \leq 1$ we obtain $\omega\left(x x^{*}\right) \omega\left(z z^{*}\right) \leq \omega\left(x^{*} x\right) \omega\left(z^{*} z\right)$. If $f(\lambda)=0$ (or $g(\lambda)=+\infty$ ) the same inequality is valid, because $x=0$ (or $z=0$ ).

Lemma 2.7. Suppose that $\omega$ is strongly 2-spectrally passive, $x \in \mathscr{A}$, $\lambda \in \boldsymbol{R}$.

(i) $\mu_{x}(\{\lambda\})=0$ if and only if $\nu_{x}(\{\lambda\})=0$.

(ii) If $\mu_{x}(\{\lambda\}) \neq 0$, then $\frac{\nu_{x}(\{\lambda\})}{\mu_{x}(\{\lambda\})}=f(\lambda)=g(\lambda)$.

Proof. Given $\lambda$ and $x$, choose $y$ in $\mathscr{A}$ as in Lemma 2.4. Then $\mu_{x}(\{\lambda\})=(x \Omega, P(\{\lambda\}) x \Omega)=\|P(\{\lambda\}) x \Omega\|^{2}=\|y \Omega\|^{2}$ (by (5)), and similarly $\nu_{x}(\{\lambda\})=\left\|P(\{-\lambda\}) x^{*} \Omega\right\|^{2}=\left\|y^{*} \Omega\right\|^{2}$ (again by (5)). Hence (i) follows because $\Omega$ is separating for $\mathscr{A}$. Moreover, if $\mu_{x}(\{\lambda\}) \neq 0$, we have

$$
g(\lambda) \leq \frac{\left\|y^{*} \Omega\right\|^{2}}{\|y \Omega\|^{2}}=\frac{\nu_{x}(\{\lambda\})}{\mu_{x}(\{\lambda\})} \leq f(\lambda),
$$

since $y \in M(-\infty, \lambda] \cap M[\lambda,+\infty)$. Then (ii) is a consequence of the fact that $f(\lambda) \leq \mathrm{g}(\lambda)$ (Lemma 2.6 (ii)).

The above lemma, taken together with Proposition 2. 1, already implies that $\Delta=f(H)$ in case $H$ has a countable spectrum. The idea of the proof of our main theorem 2.8 is that Lemma 2.7 allows us to discard arbitrary finite (even countable) subsets of $\sigma(H)$. Notice that Lemma 2.7 does not hold if $\omega$ is merely 2-spectrally passive (see Section 1).

Theorem 2.8. As before let $\mathscr{A}$ be a von Neumann algebra with cyclic and separating vector $\Omega$, and $\left\{\alpha_{i}\right\}(t \in \boldsymbol{R})$ a continuous group of *-automorphisms of $\mathscr{A}$ leaving the corresponding state $\omega$ of $\mathscr{A}$ invariant. If $\omega$ is strongly 2 -spectrally passive with respect to $\alpha$, then there exists a $P$-almost everywhere finite, decreasing function $f: \mathbb{R} \rightarrow$ $[0,+\infty]$ such that $\lrcorner=f(H)$. In fact $f$ is as defined in 2.3. 
Proof. Let $\lambda_{l}$ be the left endpoint of $\sigma(H)$ (possibly $-\infty$ ). If $\lambda>\lambda_{\ell}$, then $g(\lambda)$ is finite by Lemma 2.5 (iv), hence $f(\lambda)<+\infty$ by Lemma 2.6 (ii). For $x$ in $\mathscr{A}$ we first show that $\nu_{x} \ll \mu_{x}$ on each bounded open interval $\left(\xi, \xi^{\prime}\right)$, where $\xi>\lambda_{\ell}$. Suppose $h \in L^{1}(\boldsymbol{R})$ and $\operatorname{supp} \hat{h} \subset\left(\xi, \xi^{\prime}\right)$. Then actually supp $\hat{h} \subset\left(\xi+\varepsilon, \xi^{\prime}-\varepsilon\right)$ for some strictly positive $\varepsilon$. Put $y=\int h(t) \alpha_{t}(x) d t$, so that $y \in R\left(\xi+\varepsilon, \xi^{\prime}-\varepsilon\right) \subset M[\xi+\varepsilon$, $\left.\xi^{\prime}-\varepsilon\right]$. According to Definition 2.3 we have

$$
\begin{aligned}
g\left(\xi^{\prime}-\right) \omega\left(y^{*} y\right) & \leq g\left(\xi^{\prime}-\varepsilon\right) \omega\left(y^{*} y\right) \leq \omega\left(y y^{*}\right) \\
& \leq f(\xi+\varepsilon) \omega\left(y^{*} y\right) \leq f(\xi+) \omega\left(y^{*} y\right) .
\end{aligned}
$$

We rewrite these inequalities as

$$
g\left(\xi^{\prime}-\right) \int|\hat{h}(\lambda)|^{2} d \mu_{x}(\lambda) \leq \int|\hat{h}(\lambda)|^{2} d \nu_{x}(\lambda) \leq f(\xi+) \int|\hat{h}(\lambda)|^{2} d \mu_{x}(\lambda) .
$$

By an argument of uniform density, the above inequality holds with $|\hat{h}|^{2}$ replaced by an arbitrary nonnegative continuous function supported in $\left(\xi, \xi^{\prime}\right)$. By the Lebesgue-Radon-Nikodým theorem, $\nu_{x}$ is absolutely continuous with respect to $\mu_{x}$ on $\left(\xi, \xi^{\prime}\right)$. If $H$ is unbounded this shows at once that $\nu_{x} \ll \mu_{x}$. If $H$ is bounded, the same conclusion is obtained using Lemma 2.7 (i) with $\lambda=\lambda_{\ell}$.

We now have to prove that the Radon-Nikodým derivative $d \nu_{x} / d \mu_{x}$ is essentially independent of $x$, and that actually $d \nu_{x} / d \mu_{x}=f=g \mu_{x}-$ almost everywhere for all $x$. Fix $\xi_{0}>\lambda_{l}$ and $\varepsilon>0$. Since $0 \leq f \leq f\left(\xi_{0}\right)$ on $\left(\xi_{0},+\infty\right)$, there are only finitely many points $\xi$ in $\left(\xi_{0},+\infty\right)$ where $f(\xi-)-f(\xi+)$ exceeds a given positive value. Hence we can find a partition $\xi_{0}<\xi_{1}<\cdots<\xi_{n-1}<\xi_{n}=+\infty$ of $\left(\xi_{0},+\infty\right)$ such that

$$
f\left(\xi_{j-1}+\right)-f\left(\xi_{j}-\right)<\varepsilon \text { for } j=1,2, \ldots, n^{*}{ }^{*}
$$

On the other hand, (6) implies that for $\mu_{x}$-almost all $\lambda$ in $\left(\xi_{j-1}, \xi_{j}\right)$

$$
f\left(\xi_{j}-\right) \leq g\left(\xi_{j}-\right) \leq \frac{d \nu_{x}}{d \mu_{x}}(\lambda) \leq f\left(\xi_{j-1}+\right)
$$

(the first inequality follows from Lemma 2.6 (ii)). Since clearly

$$
f\left(\hat{\xi}_{j}-\right) \leq f(\lambda) \leq f\left(\xi_{j-1}+\right)
$$

for all $\lambda$ in $\left(\xi_{j-1}, \xi_{j}\right),(7)$ yields

*) $f\left(\xi_{n}-\right)=\lim _{\xi \rightarrow+\infty} f(\xi)$. 


$$
\left|\frac{d \nu_{x}}{d \mu_{x}}(\lambda)-f(\lambda)\right|<\varepsilon
$$

for $\mu_{x}$-almost all $\lambda$ in $\bigcup_{j=1}^{n}\left(\xi_{j-1}, \xi_{j}\right)$. By Lemma 2.7 (ii) this inequality actually holds for $\mu_{x}$-almost all $\lambda$ in $\left(\xi_{0},+\infty\right)$. Since $\varepsilon$ was arbitrary we conclude that $d \nu_{x} / d \mu_{x}=f \mu_{x}$-almost everywhere on $\left(\xi_{0},+\infty\right)$, hence $\mu_{x}$-almost everywhere on $\boldsymbol{R}$ (one has to invoke Lemma 2.7 once more if $H$ is bounded and $\lambda_{\ell}$ is an eigenvalue). Clearly a similar reasoning proves that $d \nu_{x} / d \mu_{x}=g$.

In view of the more immediate physical meaning of passivity as compared with strong spectral passivity, the following variant of the previous result may be of interest:

Corollary 2.9. Adopting the same general assumptions as in Theorem 2. 8, suppose that the following properties hold for the triple $(\mathscr{A}, \alpha, \omega):$

(i) $\omega$ is 2-spectrally passive with respect to $\alpha$.

(ii) $H$ has continuous spectrum except at 0 (i.e. 0 is the only eigenvalue of $H$, or $M(\{\lambda\})=\{0\}$ whenever $\lambda \neq 0)$.

(iii) For all $x$ in $\mathscr{A}$,

$$
\lim _{T \rightarrow \infty} \frac{1}{2 T} \int_{-T}^{T} \omega\left(\left[x^{*}, \alpha_{t}(x)\right]\right) d t=0 .
$$

Then $J=f(H)$ for some decreasing, P-essentially finite function $f$ : $\boldsymbol{R} \rightarrow[0,+\infty]$.

This corollary follows from Theorem 2. 8 and the following lemma.

Lemma 2.10. If $\omega$ is n-spectrally passive with respect to $\alpha$, and the above conditions (ii) and (iii) hold, then $\omega$ is strongly n-spectrally passive.

Proof. First assume that $\omega$ is spectrally passive and $x \in M[0,+\infty)$. Writing $x=y+z$ with $y \in M(\{0\})$ and $z \in R(0,+\infty)$ (Lemma 2.4(i)), we have $\|x * \Omega\|^{2}=\left\|y^{*} \Omega\right\|^{2}+\left\|z^{*} \Omega\right\|^{2}$ because $y^{*} \Omega$ and $z^{*} \Omega$ are orthogonal. But $\left\|y^{*} \Omega\right\|^{2}=\|y \Omega\|^{2}$ by (iii) and Lemma 2.4 (iii), and $\left\|z^{*} \Omega\right\|^{2} \leq\|z \Omega\|^{2}$ by assumption. Hence

$$
\|x * \Omega\|^{2} \leq\|y \Omega \mid\|^{2}+\|z \Omega\|^{2}=\|x \Omega\|^{2} .
$$


This shows that $\omega$ is strongly spectrally passive.

Now it is easy to set up an induction proof. Suppose the lemma holds for $n=m-1$, and $x_{i} \in M\left[\lambda_{i},+\infty\right)(i=1,2, \ldots, m), \sum_{i=1}^{m} \lambda_{i} \geq 0$. By (ii) and Lemma 2.4 (i), $M[\lambda,+\infty)=R(\lambda,+\infty)$ except possibly if $\lambda=0$. Hence if $\lambda_{i} \neq 0$ for all $i$, the inequality $\prod_{i=1}^{m} \omega\left(x_{i} x_{i}^{*}\right) \leq \prod_{i=1}^{m} \omega\left(x_{i}^{*} x_{i}\right)$ follows from the assumption of $m$-spectral passivity. If $\lambda_{1}=0$, say, then $\underset{m}{\omega}\left(x_{1} x_{1}^{*}\right) \leq \omega\left(x_{1}^{*} x_{1}\right)$ by the first part of the proof and $\prod_{i=2}^{m} \omega\left(x_{i} x_{i}^{*}\right) \leq$ $\prod_{i=2}^{m} \omega\left(x_{i}^{*} x_{i}\right)$ by the induction hypothesis. Multiplying these inequalities we conclude that $\omega$ is strongly $m$-spectrally passive.

Remark 2.11. The condition (iii) in the statement of Corollary 2.9 is easily seen to be equivalent with

$$
\mu_{x}(\{0\})=\nu_{x}(\{0\}) \text { for all } x \in \mathscr{A},
$$

and is thus a necessary condition for the equality $\Delta=f(H)$ to hold. One can say that it amounts to "the KMS condition at 0 energy". This formulation is of ten used to describe the main direct consequence of the stability notion due to Haag, Kastler and Trych-Pohlmeyer (see [9, pp. 177-178]).

Concluding this section, we prove that strong 2-spectral passivity is not only a sufficient but also a necessary condition for the equation $\Delta=f(H)$ to hold (with decreasing $f$ ).

Theorem 2. 12. If $\Delta=f(H)$, where $f: \boldsymbol{R} \rightarrow[0,+\infty]$ is everywhere decreasing, then $\omega$ is strongly 2-spectrally passive.

Proof. First we observe that $\Delta=f(H)$ implies that $f(-\lambda)=f(\lambda)^{-1}$ for $P$-almost all $\lambda$. This follows from the fact that

$$
f(H)^{-1}=\Delta^{-1}=J \Delta J=J f(H) J=f(-H),
$$

where $J$ has the usual meaning (i. e. $J \Delta^{1 / 2} x \Omega=x^{*} \Omega$ for all $x$ in $\mathscr{A}$ ), and the last equality follows from $J H J=-H$. In particular $f(0)=1$.

As before put $\lambda_{\ell}=\inf \sigma(H)$. If $\sigma(H)=\{0\}$ then $\Delta=1$ and $\omega$ is a trace, so we can suppose $\lambda_{\ell}<0$. If $\lambda \in\left(\lambda_{\ell},-\lambda_{\ell}\right)$ then $0<f( \pm \lambda)<+\infty$, where the first inequality follows from the injectivity of $\Delta$. Let us write $f_{e}(-\lambda)$ for the $P$-essential supremum of $\{f(\xi) \mid \xi \in[-\lambda,+\infty)\}$. 
If $\xi \geq-\lambda$, then $f(-\xi)^{-1} \leq f(\lambda)^{-1}$ because $f$ is decreasing, hence by the above observation $f(\xi) \leq f(\lambda)^{-1}$ for $P$-almost all $\xi$ in $[-\lambda,+\infty)$. It follows that $f_{e}(-\lambda) \leq f(\lambda)^{-1}$. Thus for $x$ in $M[\lambda,+\infty)$ and $y$ in $M[-\lambda,+\infty)$ we obtain

$$
\begin{aligned}
\omega\left(x x^{*}\right) \omega\left(y y^{*}\right) & =\left\|\Delta^{1 / 2} x \Omega\right\|^{2}\left\|\Delta^{1 / 2} y \Omega\right\|^{2} \\
& =\int f(\xi) d \mu_{x}(\xi) \cdot \int f(\xi) d \mu_{y}(\xi) \\
& \leq f(\lambda) \omega\left(x^{*} x\right) \cdot f_{e}(-\lambda) \omega\left(y^{*} y\right) \text { because supp } \mu_{x} \\
& \quad \subset[\lambda,+\infty), \operatorname{supp} \mu_{y} \subset[-\lambda,+\infty) \quad[7, \text { Lemma } 1.6] \\
& \leq \omega\left(x^{*} x\right) \omega\left(y^{*} y\right) .
\end{aligned}
$$

This shows that $\omega$ is strongly 2-spectrally passive, at least if $H$ is unbounded. If $H$ is bounded we also have to consider $x$ in $M\left[-\lambda_{\ell}\right.$, $+\infty)$ and $y$ in $M\left[\lambda_{\ell},+\infty\right)=\mathscr{A}$. Then either $\pm \lambda_{\ell}$ are eigenvalues of $H$, in which case $0<f\left( \pm \lambda_{\ell}\right)<+\infty$ and the previous reasoning still goes through (with $-\lambda_{\ell}$ in place of $\lambda$ ); or $\pm \lambda_{\ell}$ are not eigenvalues cf $H$, and then $M\left[-\lambda_{\ell},+\infty\right)=\{0\}$.

Remark 2.13. Theorem 2.8 can be generalized to deal with a not necessarily faithful strongly 2 -spectrally passive state $\omega$. Let $\mathscr{A}$ be represented in the corresponding GNS representation, and let $Q$ denote the support of $\omega$, i. e. the projection onto the closure of $\mathscr{A}^{\prime} \Omega$. For $\lambda$ in $\boldsymbol{R}$ define

$$
\begin{aligned}
& f(\lambda)=\inf \left\{a \geq 0 \mid \omega\left(x x^{*}\right) \leq a \omega\left(x^{*} x\right) \quad \text { for all } x \text { in } M[\lambda,+\infty)\right\} \\
& g(\lambda)=\sup \left\{b \geq 0 \mid \omega\left(x x^{*}\right) \geq b \omega\left(x^{*} x\right) \quad \text { for all } x \text { in } M(-\infty, \lambda]\right\}
\end{aligned}
$$

(these prescriptions coincide with Definition 2.3 if $\omega$ is faithful).

We make the following observations:

(i) If $f(\lambda)=0$ then $Q \leq P(-\infty, \lambda)$. Indeed, if $x \in M[\lambda,+\infty)$ and $y \in \mathscr{A}^{\prime}$ then $(y \Omega, x \Omega)=\left(y x^{*} \Omega, \Omega\right)=0$ because $x * \Omega=0$. Hence $Q x \Omega$ $=0$. Using Lemma 2.4 (i) it follows that $Q P[\lambda,+\infty)=0$.

(ii) If $g(\lambda)>0$ then $Q \geq P(-\infty, \lambda]$. To see this, consider $x$ in $M(-\infty, \lambda]$. Clearly $(1-Q) x \in M(-\infty, \lambda]$ and $x^{*}(1-Q) \Omega=0$. Then $(1-Q) x \Omega=0$ because $g(\lambda)>0$. Again using Lemma 2.4 (i) we conclude that $(1-Q) P(-\infty, \lambda]=0$.

Now we put $\mu=\inf \{\lambda \mid f(\lambda)=0\} \in \boldsymbol{R} \cup\{+\infty\}$. By (i) $Q \leq P(-\infty$, $\mu]$, and $Q \leq P(-\infty, \mu)$ if $f(\mu)=0$. The assumption of strong 2spectral passivity implies $f \leq g$ (as in Lemma 2.6). Hence by (ii) 
$Q \geq P(-\infty, \mu)$, and $Q \geq P(-\infty, \mu]$ if $f(\mu)>0$. We conclude that either $Q=P(-\infty, \mu)$ (if $f(\mu)=0$ ) or $Q=P(-\infty, \mu]$ (if $f(\mu)>0)$.

If we define $\Delta$ as the direct sum of the usual modular operator associated to the cyclic and separating vector $\Omega$ of the von Neumann algebra $Q \mathscr{A} Q$ on $Q \mathscr{H}$ and the zero-operator on $(1-Q) \mathscr{H}$ (as is done e.g. in [12, p. 284]), then it is still true that $\Delta=f(H)$. Notice also that if $\omega$ is not faithful $(\mu<\infty)$ then $H$ is bounded below (by $-\mu$ ).

\section{§3. Equilibrium and 3-Passivity}

If a faithful invariant state $\omega$ of a $W^{*}$-dynamical system is known to be strongly 2-spectrally passive, so that $\Delta=f(H)$ by Theorem 2.8, then in order to decide whether $\omega$ is $\beta-\mathrm{KMS}$ it is of course sufficient to check if $f(\lambda)=e^{-\beta \lambda}$ for $P$-almost all $\lambda(0 \leq \beta<+\infty)$.

Since the KMS condition is known to follow from complete spectral passivity [7, Theorem 1.3], it is natural, as a first step, to study the implications of 3-spectral passivity on the properties of $f$. Since our final result is obtained under the assumption that $\sigma(H)=\boldsymbol{R}$, we can restrict our attention at once to the case where $H$ is unbounded. This is convenient technically because it implies $0<f, g<+\infty$.

Lemma 3.1. Let $f$ and $g$ be as in Definition 2.3, and suppose $H$ is unbounded. Then the following are equivalent:

(i) $\omega$ is strongly 3-spectrally passive.

(ii) For all $\lambda, \lambda^{\prime}$ in $\boldsymbol{R}$,

(iii) For all $\lambda, \lambda^{\prime}$ in $\boldsymbol{R}$,

$$
f(\lambda) f\left(\lambda^{\prime}\right) \leq g\left(\lambda+\lambda^{\prime}\right) \text {. }
$$

$$
f\left(\lambda+\lambda^{\prime}\right) \leq g(\lambda) g\left(\lambda^{\prime}\right) .
$$

Proof. Straightforward, and left to the reader (compare with Lemma 2.6).

Next we wish to turn the inequalities (ii) and (iii) above into equalities. To this end we make the assumption that $\sigma(H)=\boldsymbol{R}$, which implies that $g\left(\lambda_{+}\right) \leq f\left(\lambda_{-}\right)$for all $\lambda$ in $\boldsymbol{R}$ (Lemma $2.5(\mathrm{v})$ ). Let $D$ be the (finite or countable) set of those points in $\boldsymbol{R}$ where at least one of the functions $f$ and $g$ is discontinuous. If none of the 
points $\lambda, \lambda^{\prime}, \lambda+\lambda^{\prime}$ belongs to $D$, one has, under the assumption of strong 3-spectral passivity (Lemma 3.1 and $2.5(\mathrm{v})$ ):

$$
f(\lambda) f\left(\lambda^{\prime}\right) \leq g\left(\lambda+\lambda^{\prime}\right) \leq f\left(\lambda+\lambda^{\prime}\right)
$$

and

$$
f\left(\lambda+\lambda^{\prime}\right) \leq g(\lambda) g\left(\lambda^{\prime}\right) \leq f(\lambda) f\left(\lambda^{\prime}\right)
$$

i. e.

$$
f(\lambda) f\left(\lambda^{\prime}\right)=f\left(\lambda+\lambda^{\prime}\right)
$$

provided $\lambda \notin D, \lambda^{\prime} \notin D, \lambda+\lambda^{\prime} \notin D$.

Let $E$ be the set of all real numbers $\lambda$ for which there exists a number $\phi(\lambda)$ such that $f(\lambda+\mu) f(\mu)^{-1}=\phi(\lambda)$ for all $\mu$ outside some finite or countable subset of $\boldsymbol{R}$. Clearly $E$ is an additive semigroup and

$$
\phi(\lambda) \phi\left(\lambda^{\prime}\right)=\phi\left(\lambda+\lambda^{\prime}\right)
$$

whenever $\lambda, \lambda^{\prime} \in E$. Moreover the complement of $E$ is contained in $D$ by $(10)$, and $\phi(\lambda)=f(\lambda)$ if $\lambda \notin D$. It follows easily that $E=\mathbb{R}$ and that $\phi$ is Borel measurable (because $f$ is). Consequently $\phi(\lambda)=e^{-\beta \lambda}$ for all $\lambda$ in $\boldsymbol{R}$, where $\beta \geq 0$.

It remains to be shown that $f(\lambda)=e^{-\beta \lambda}$ for all $\lambda$ in $\mathbb{R}$. Suppose $f(\lambda)>e^{-\beta \lambda}$ for some $\lambda$. Then there exists a positive number $\delta$ such that $e^{-\beta \mu}<f(\lambda)$ whenever $\lambda-\delta \leq \mu \leq \lambda$, and hence $e^{-\beta \mu}<f(\mu)$ since $f$ decreases. But this contradicts the fact that $f(\mu)=\phi(\mu)=e^{-\beta \mu}$ for all but at most countably many values of $\mu$. The possibility that $f(\lambda)<$ $e^{-\beta \lambda}$ is ruled out in a similar way. As $\sigma(H)=s p(\alpha)$ [7] we have shown:

Theorem 3.2. If $s p(\alpha)=\boldsymbol{R}$, then any faithful, normal, strongly 3-spectrally passive state of $(\mathscr{A}, \alpha)$ is $\beta$-KMS with respect to $\alpha$ for some $\beta, 0 \leq \beta<+\infty$.

Remark 3.3. After this work was finished it was pointed out to the author by H. Daniëls that the result of Theorem 3.2 still holds if the assumption of strong 3-spectral passivity is replaced by 3-spectral passivity. This is seen by combining [6b, Theorem 1.23] with [7, Theorem 3.3]. In fact one can also easily adapt the above proof to accomodate this more general situation. It is sufficient to redefine $f$ and $g$ as

$$
f(\lambda)=\sup \left\{\|x * \Omega\|^{2} \|\left. x \Omega\right|_{1} ^{\mid-2} \mid x \in R(\lambda,+\infty), x \neq 0\right\}
$$

and

$$
g(\lambda)=\inf \left\{\left\|x^{*} \Omega\right\|^{2}\|x \Omega\|^{-2} \mid x \in R(-\infty, \lambda), x \neq 0\right\} \text {. }
$$


It is still true that $\lambda \in \sigma(H)$ implies $g(\lambda+) \leq f(\lambda-)$, and exactly as before one shows that $f(\lambda)=e^{-\beta \lambda}$ for some $\beta$. It follows that $\omega$ is KMS by [7, Theorem 1.1].

These results should be compared with the counterexample 4.9 in [7] of an $n$-spectrally passive state that is not $(n+1)$-spectrally passive. Let us finally point out that in classical mechanics (under suitable regularity assumptions) 2-passivity is already sufficient to ensure equilibrium [8, Theorems 1 and $2 ; 6 \mathrm{~b}$, Theorem 3.19].

\section{Acknowledgments}

I would like to thank $\mathrm{H}$. Araki for remarks leading to an improvement of the original version of Lemma 2.4, H. Daniëls for sending me his 1982 Groningen thesis, and A. Ramsay for a useful conversation concerning the proof of Theorem 3.2.

\section{References}

[1] Araki, H., Relative Hamiltonian for faithful normal states of a von Neumann algebra, Publ. R.I.M.S. Kyoto Univ., 9, (1973), 165-209.

[2] - Relative entropy of states of von Neumann algebras, Publ. R.I.M.S. Kyoto Univ., 11, (1976), 809-833.

[3] Arveson, W. B., On groups of automorphisms of operator algebras, J. Funct. Anal., 15, (1974), 217-243.

[4] Batty, C. J. K., The KMS condition and passive states, J. Funct. Anal., 46, (1982), 246257.

[5] The KMS condition and spectral passivity in group duality, Preprint, $17 \mathrm{pp}$. (1982).

[6a] Daniëls, H. A. M., Passivity and equilibrium for classical Hamiltonian systems, J. Math. Phys., 22, (1981), 843-846.

[6b] - Passivity and equilibrium for thermodynamical systems, Thesis, University of Groningen (1982).

[7] De Cannière, J., A spectral characterization of KMS states, Commun. Math. Phys., 84, 4, (1982), 187-205.

[8] Gorecki, J. and Pusz, W., Passive states for finite classical systems, Lett. Math. Phys, 4, (1980), 433-443.

[9] Haag, R., Kastler, D. and Trych-Pohlmeyer, E. B., Stability and equilibrium states, Commun. Math. Phys., 38, (1974), 173-193.

[10] Lenard, A., Thermodynamical proof of the Gibbs formula for elementary quantum systems, J. Stat. Phys, 19, (1978), 575-586.

[11] Olesen, D., On spectral subspaces and their application to automorphism groups, Symp. Math., 20, (1976), 253-296.

[12] Pusz, W. and Woronowicz, S. L., Passive states and KMS states for general quantum systems, Commun. Math. Phys., 58, (1978), 273-290.

[13] Takesaki, M., Tomita's theory of modular Hilbert algebras and its applications, Lecture Notes in Mathematics 128, Berlin, Heidelberg, New York, Springer 1970. 